\title{
VULNERABILIDAD, PROTECCIÓN Y ASISTENCIA SOCIAL EN EL ADULTO MAYOR EN MÉXICO
}

Rosario Guadalupe Hernández de Dios, ${ }^{1}$ Martín Francisco Montaño Hernández ${ }^{2}$ y Manuel Zavaleta Suárez. ${ }^{3}$

Resumen. Actualmente se han agudizado las condiciones de vulnerabilidad de los grupos sociales más desprotegidos como son en este caso las personas adultas mayores en condiciones de soledad, abandono, discriminación, discapacidad, así como víctimas del maltrato en sus diversas expresiones o los riesgos asociados a la protección social. Observando las tendencias del incremento poblacional que registra cerca de 10.5 millones de adultos mayores en México, lo que representa 9.0\%, es decir, casi 1 de cada 10 habitantes del país, por lo tanto es evidente la demanda de la asistencia social.

Palabras clave: vulnerabilidad, Adultos mayores, condiciones, riesgos, población.

Abstract. Currently are exacerbating the vulnerability of the most vulnerable social groups, such as in this case the elderly in conditions of loneliness, abandonment, discrimination, disabilities and victims of abuse in its various expressions or risks associated with protection Social. Noting the trends of population growth that registers about 10.5 million older adults in Mexico, representing 9.0\%, in almost 1 in 10 people in the country, therefore it is clear the demand for social assistance

Keywords: vulnerability, elderly, conditions, risks, population

\footnotetext{
${ }^{1}$ Doctora en Ciencias Administrativas, por la Facultad de Ciencias Administrativas de la UABC; Perfil Deseable PRODEP desde 2014-2017, Profesor Investigador del Cuerpo Académico: Ciencias Económicas y Sociales, Investigador Responsable de Proyectos de Investigación (2016-2017) en la Facultad de Ciencias Sociales y Políticas UABC.

2 Maestro en Administración, por la Facultad de Ciencias Administrativa de la UABC; cuenta con el reconocimiento al Perfil Deseable del Programa para el Desarrollo Profesional Docente (PRODEP de la SEP, desde 2015), ha impartido los cursos de Entorno Económico Internacional, Finanzas Internacionales, Formulación y Evaluación de Proyectos de Inversión, Facultad de Ciencias Administrativas, UABC.

${ }^{3}$ Doctor en Ciencias Administrativas, por la Facultad de Ciencias Administrativas de la UABC; cuenta con el reconocimiento al Perfil Deseable del Programa para el Desarrollo Profesional Docente (PRODEP de la SEP) Actualmente es coordinador de posgrado e Investigación en la FCSyP UABC.
} 


\section{Introducción}

Actualmente se han agudizado las condiciones de vulnerabilidad de los grupos sociales más desprotegidos como son en este caso las personas adultas mayores en condiciones de soledad, abandono, discriminación, discapacidad, así como víctimas del maltrato en sus diversas expresiones o los riesgos asociados a la protección social. Observando las tendencias del incremento poblacional que registra cerca de 10.5 millones de adultos mayores en México, lo que representa $9.0 \%$, es decir, casi 1 de cada 10 habitantes del país, por lo tanto, es evidente la demanda de la asistencia social. (INEGI, 2010).

En el año 2010, las entidades federativas con los mayores montos de población de 60 y más años de edad del país eran el estado de México, el Distrito Federal, Veracruz, Jalisco y Puebla, con un monto que va de poco más de 514 mil a 1.1 millones de habitantes. Las cinco entidades agrupan a 4.1 millones de adultos mayores, en otras palabras, poco más del $40.0 \%$ de dicha población. (Gráfica 1).

Asimismo, se observa que las mayores tasas de crecimiento promedio anual para el periodo 2000-2010 corresponden a Quintana Roo (6.8\%), Baja California Sur (5.3\%), Baja California (5.0 por ciento); y en menor medida, Querétaro, Chiapas y el estado de México.

\section{Vulnerabilidad}

Se define y se toma como factor de riesgo interno de un sujeto o sistema expuesto a una amenaza, correspondiente a su predisposición específica a ser afectado o ser susceptible a sufrir daño; se refiere al ámbito físico, económico, político o social, lo cual ocasiona desestabilización. Por otro lado, Susman (1984), refiere a la vulnerabilidad como el grado en que las diversas clases sociales están diferencialmente en riesgo. 
Se ve la vulnerabilidad como falta de capacidad para defenderse y superar una calamidad (Chambers, 1989). Se refiere a las regiones, ya que se presenta la denigración por pobreza propensa por desastres (Bankoff, 2001).

"La definición básica de vulnerabilidad se refiere a las características de una persona o grupo y su situación, que influencian su capacidad de anticipar, lidiar, resistir y recuperarse del impacto de una amenaza". (Wisner, 2004).

“Al definir vulnerabilidad como un proceso de pérdida, se implica que:

- Existe una situación previa ante la cual hay una transformación y

- Hay una evaluación de dicha transformación respecto a un parámetro de lo 'normal' o 'positivo', definido teórica y metodológicamente". (Ruiz, 2012).

"Como menciona Alwanget, 2003 distingue entre vulnerabilidad a (situaciones sociales que resultan de procesos de pérdida) y vulnerabilidad ante (amenazas y situaciones de estrés que cambian las condiciones de la unidad de análisis en un tiempo específico.”

“En la especificación de los marcos teóricos y metodológicos adecuados para identificar quiénes son vulnerables y cuál es la intensidad de la vulnerabilidad, un punto clave es especificar ante qué peligros, eventos, procesos o fenómenos se es vulnerable" (Wisner, 2004).

Por otro lado, Moser (1998) la utiliza para analizar a los pobres, las características de sus hogares y su entorno próximo, como las herramientas-instrumentos para enfrentar o salir de la pobreza. “...debilidad objetiva de los pobres (vulnerabilidad) para enfrentar la sobrevivencia cotidiana, o con mayor razón, las crisis económicas, podrían ser contrarrestadas con una adecuada gestión de los activos que tienen con independencia de sus 
ingresos escasos..." (Citado por Rodríguez Vignoli, 2001:18). Este enfoque vincula estrechamente vulnerabilidad con pobreza.

Analizando la hipótesis de la vulnerabilidad y carencias de desarrollo Cardona (2001), propone los siguientes factores, los cuales la originan;

Fragilidad física o exposición. Se refiere a la situación de susceptibilidad que tiene el asentamiento humano de ser afectado o por estar, en áreas de influencia de fenómenos peligrosos y falta de resistencia física.

Fragilidad social. Es la predisposición que surge como resultado al nivel de marginalidad y segregación social de asentamientos humanos en condiciones de desventaja y debilidad relativa por factores socioeconómicos.

$\underline{\text { Falta de resiliencia }}^{4}$. Es la limitación de acceso y movilidad de recursos del asentamiento humano, incapacidad de respuesta, así como deficiencias para absorber el impacto.

\section{Vulnerabilidad en el Adulto Mayor}

En México como en muchos países del mundo, el envejecimiento poblacional ha tomado mayor importancia en los últimos años; las personas en edad avanzada conforman uno de los grupos con mayor vulnerabilidad, misma que se incrementa si tienen alguna limitación, por ello conocer las características sociodemográficas de las personas en edad de 60 y más años con limitación en la actividad es importante para proponer políticas públicas para favorecer este sector poblacional. (Gráfica 1 y Cuadro 1).

\footnotetext{
${ }^{4}$ Definición. La resiliencia es un proceso dinámico, constructivo, de origen interactivo, sociocultural que conduce a la optimización de los recursos humanos y permite sobreponerse a las situaciones adversas. Se manifiesta en distintos niveles del desarrollo, biológico, neurofisiológico y endocrino en respuesta a los estímulos ambientales. Kotliarenco, María Angélica y Cáceres, Irma. (2011).
} 
Dado que las personas de 60 y más años se consideran un grupo vulnerable por ser adultos en una de las últimas etapas de la vida y aún más por tener limitación en la actividad y ser susceptibles de cuidados específicos. Ahora bien ¿Cuánta población adulta mayor se encuentra en estado Vulnerable?

Los adultos mayores con alguna limitación en la actividad, crecieron poco más del triple en el censo 2010 con 2.8 millones lo que representa 26.3 por ciento en comparación del 2000 con un registro de 745 mil casos representando $10.7 \%$ de adultos de 60 años y más. (INEGI, 2010).

Acorde a las cifras presentadas por el Censo de Población y Vivienda 2010, de la población adulta mayor, $4.3 \%$ presenta al menos una limitación, (Gráfica 2), es decir 1.7 millones de los adultos mayores. Mientras que $0.5 \%$ registra tener 2 o más limitaciones, lo que representa 197 mil casos. 
Gráfica 1. Población de 60 y más años por entidad federativa y tasa de crecimiento promedio anual 2000-2010.

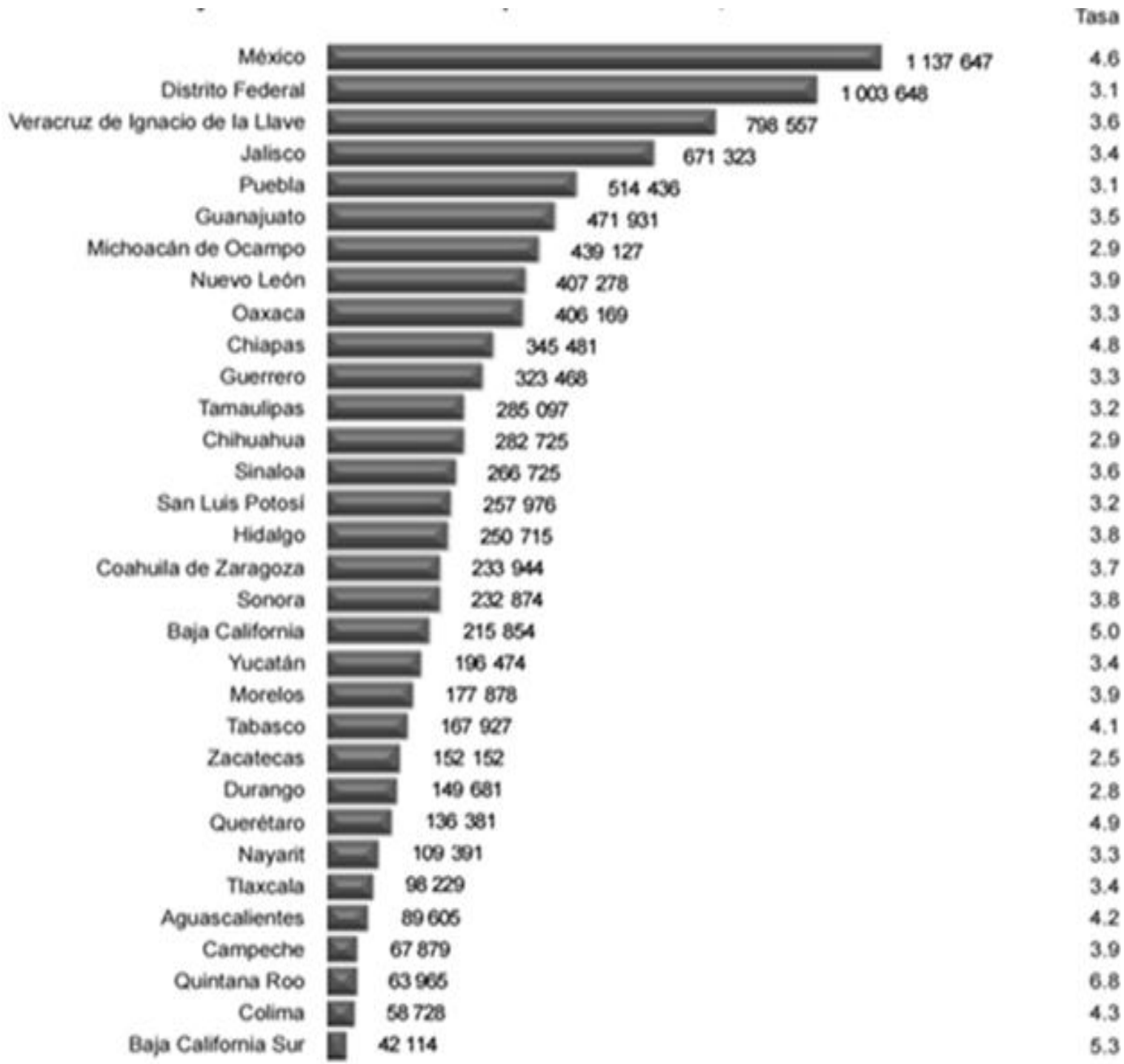

Fuente: INEGI. XII Censo de Población y Vivienda 2000; Censo de Población y Vivienda 2010. 
Cuadro 1. Porcentaje de la población de 60 y más años por grupos de edad según condición de limitación en la actividad y relación hombres-mujeres, 2000 y 2010.

\begin{tabular}{|c|c|c|c|c|c|c|}
\hline \multirow[b]{2}{*}{ Grupos de edad } & \multirow[t]{2}{*}{ Total } & \multicolumn{2}{|c|}{ Con limitación } & \multicolumn{2}{|c|}{ Sin limitación } & \multirow{2}{*}{$\begin{array}{r}\text { No } \\
\text { especificado }\end{array}$} \\
\hline & & Población & $\begin{array}{r}\text { Relación } \\
\text { hombres-mujeres }\end{array}$ & Población & $\begin{array}{r}\text { Relación } \\
\text { hombres-mujeres }\end{array}$ & \\
\hline 2010 & 100.0 & 26.3 & 80 & 73.3 & 90 & 0.4 \\
\hline 60 a 64 anos & 100.0 & 14.6 & 84 & 85.0 & 92 & 0.4 \\
\hline 65 a 84 anos & 100.0 & 28.4 & 82 & 71.1 & 90 & 0.5 \\
\hline 85 y más anos & 100.0 & 58.7 & 69 & 40.8 & 82 & 0.5 \\
\hline 2000 & 100.0 & 10.7 & 89 & 88.7 & 88 & 0.6 \\
\hline 60 a 64 años & 100.0 & 5.3 & 107 & 94.3 & 90 & 0.4 \\
\hline 65 a 84 antos & 100.0 & 11.3 & 93 & 88.2 & 88 & 0.5 \\
\hline 85 y más años & 100.0 & 30.0 & 69 & 69.1 & 76 & 0.9 \\
\hline
\end{tabular}

Fuente: INEGI. XII Censo General de Población y Vivienda 2000, cuestionario básico; Censo de Población y Vivienda 2010.

Gráfica 2. Porcentaje de la población de 60 y más años de edad con limitación en la actividad según número de limitaciones, 2010.

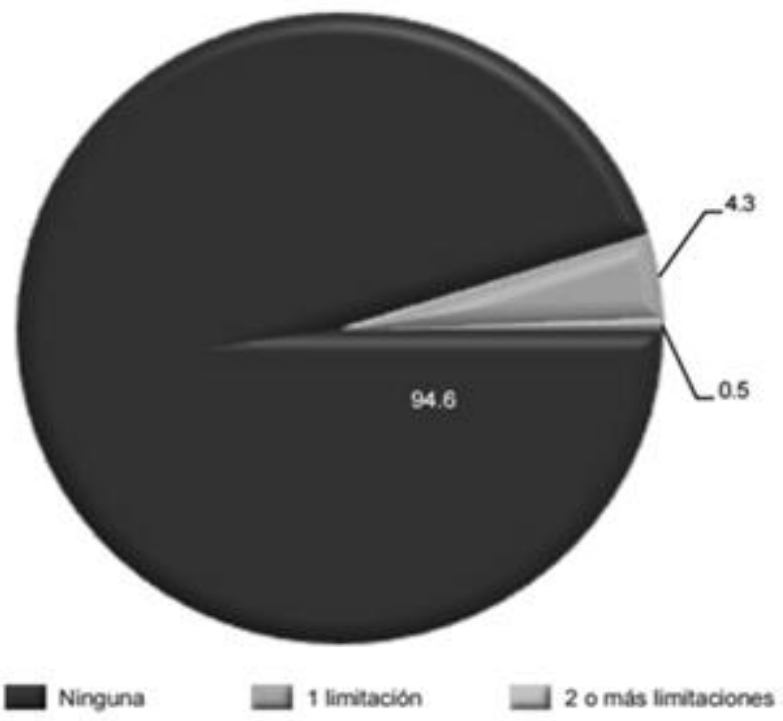

Notac La suma de los porcentajes puede no sumar $100 \%$, debido a que no se grafica el valor del no especificado.

Fuente: INEGI. Censo de Población y Vivienda 2010, cuestionario ampliado. 
Referente a protección social, protección de la seguridad económica de las personas y sus hogares ante eventos de la vida, como el desempleo, la enfermedad, la invalidez, la muerte y la vejez. También se considera las limitaciones que ciertos hogares tienen para obtener un ingreso suficiente para adquirir una canasta básica alimentaria.

Por otro lado, se observa que 17 estados o entidades federativas se encuentran por encima del promedio nacional, las entidades con el mayor valor porcentual de la población de 60 y más años con al menos una limitación en la actividad son Tabasco 5.5\% que representa 43 mil casos, Durango 5.4\% que equivale a 29 mil, Coahuila 5.3\% que corresponde a 53 mil adultos en esa situación. En el otro extremo se muestra a Nuevo León 3.0\% que significa 53 mil registros; Baja California y Quintana Roo, ambas con el 3.5\% y representa 40 y 17 mil adultos respectivamente con alguna limitación. (INEGI. Censo de Población y Vivienda 2010).

\section{Protección Social}

Este término se toma como punto focal la protección de la seguridad económica de las personas y sus hogares ante eventos de la vida, como son el desempleo, la enfermedad, la invalidez, la muerte y la vejez. ${ }^{5}$

El Banco Mundial propone como "marco conceptual para protección social el manejo social de riesgos y la describe como las intervenciones públicas para: a) ayudar a individuos, hogares y comunidades a manejar mejor el riesgo, y b) proveer apoyo a los pobres" (Holzmann y Jorgensen, 2010).

\footnotetext{
${ }^{5}$ Esta definición retoma los principios filosóficos con que nace la seguridad social. Se refiere exclusivamente a ciertos riesgos idiosincráticos. Ello no implica que el Estado no deba tener programas y acciones para prevenir y atender otros riesgos idiosincráticos, así como los de carácter sistemático. El concepto de seguridad económica refiere la protección ante fluctuaciones del consumo de los hogares.
} 
En la última década se observa una literatura creciente sobre protección social y un consenso de que, en países en desarrollo, la protección social es una respuesta efectiva a la pobreza y la vulnerabilidad y un componente esencial en las estrategias de desarrollo económico y social. Estudios recientes han argumentado que la noción de la seguridad social es muy limitada dadas las necesidades sociales y económicas de los países en desarrollo y que debería usarse una noción más extensiva de protección social (Kabeer, 2002; Barrientos y Shepherd, 2003).

La Organización Internacional del Trabajo (OIT) define la seguridad social como "la protección que la sociedad provee para sus miembros a través de una serie de medidas públicas contra el sufrimiento social y económico que de otra manera serían causados por la ausencia o reducción sustantiva en los ingresos como resultado de la enfermedad, maternidad, accidentes laborales, invalidez y muerte; la provisión de cuidado médico; y la provisión de subsidios para familias con niños" (OIT, 1984). Por lo tanto, el surgimiento de los Derechos Humanos.

Los riesgos asociados a la protección social en el transcurso del ciclo de vida, y que se analizarán en esta evaluación, se representan en el cuadro 2. En las filas se muestran los que tienen que ver con cuatro dimensiones clave de la protección social: a) acceso a la salud; b) el trabajo; c) la orfandad, discapacidad y vejez; y d) el ingreso. (CONEVAL). 


\section{Cuadro 2. Principales riesgos asociados a la protección social en el curso de vida. ${ }^{6}$}

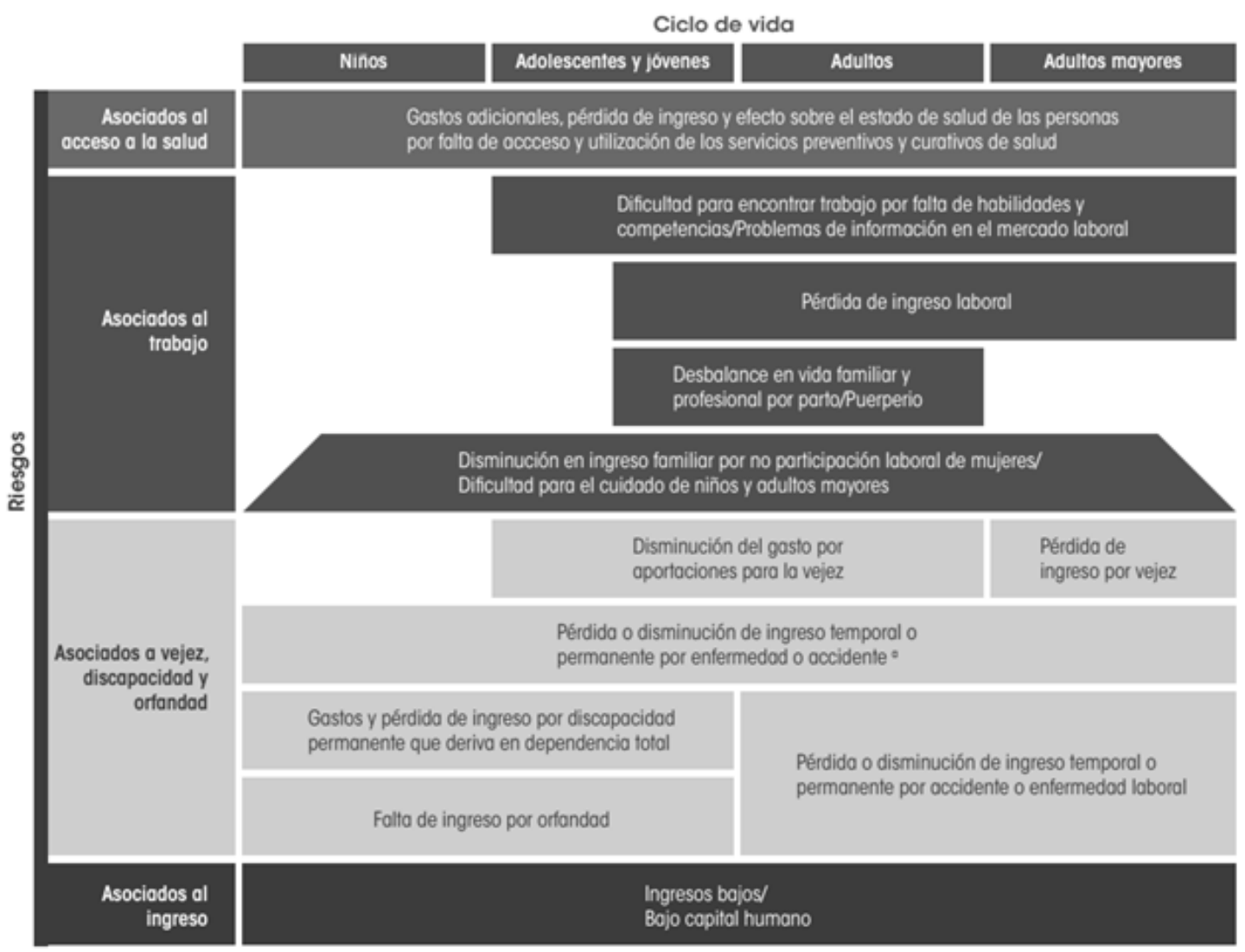

Fuente: CONEVAL

En las columnas se representan cuatro grupos etarios de acuerdo con diferentes momentos del ciclo de vida: niños, adolescentes y jóvenes, adultos y adultos mayores. Las celdas

\footnotetext{
${ }^{6}$ Se consideran situaciones en las que una enfermedad o accidente imposibilita a las personas a realizar una actividad económica remunerada, tales como ceguera, sordera, parálisis o falta de un miembro específico, entre otros. En el caso de los menores de edad, este riesgo se refiere a la situación en la que éstos tienen una discapacidad o una enfermedad que exige que un integrante del hogar deje de trabajar para dedicarse a su cuidado; o bien, que alguien que podría contribuir al gasto doméstico deje de hacerlo para ocuparse de su cuidado.
} 
simbolizan los riesgos relacionados con una de las dimensiones de protección social que enfrentan los individuos que se encuentran en un momento del ciclo de vida particular.

En la celda verde indica que todos los individuos a lo largo de su ciclo de vida pueden enfrentar gastos adicionales, pérdida de ingreso y tener un efecto sobre su estado de salud debido a falta de acceso efectivo de los servicios preventivos de salud. En la celda azul señala que tanto los adolescentes y jóvenes como los adultos y adultos mayores pueden enfrentarse a la dificultad para encontrar trabajo, sea por falta de habilidades y competencias o por problemas de información en el mercado laboral. En la celda gris se tienen las pérdidas asociadas a vejez, discapacidad y orfandad, o disminución de ingreso temporal o permanente por accidente o enfermedad laboral, falta de ingreso por oportunidad, pérdida de ingresos por vejez o disminución del gasto por aportaciones para la vejez.

Por un lado, mientras la Población Económicamente Activa (PNEA) pensionada o jubilada y la dedicada a los quehaceres del hogar realizan una menor contribución con el paso de los años; por el otro, las limitaciones físicas o mentales que se acentúan (Gráfica 3). Se supone que en este trayecto merman las capacidades y habilidades motrices, situación que imposibilita la participación en los quehaceres del hogar. 
Gráfica 3. Distribución porcentual de la población de 60 y más años no económicamente activa por grupos quinquenales de edad según tipo de actividad no

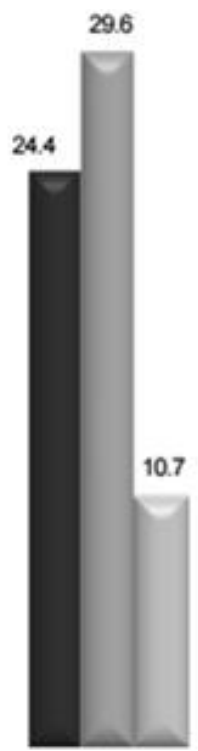

$60-64$ anos

Pensionada o Jubilada económica, 2010.

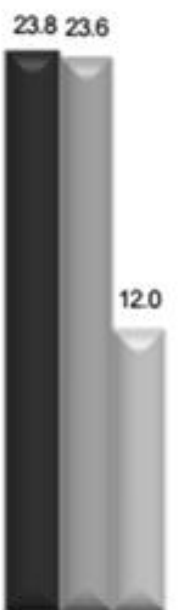

65.69 anos

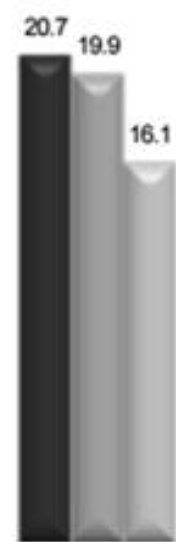

$70-74$ anos

Se dedica a los quehaceres del hogar

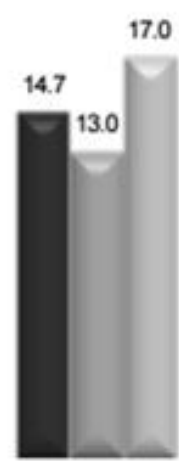

75-79 anos

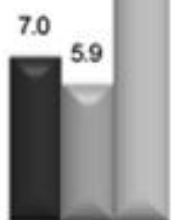

85 y más anos

Tiene alguna limitacion fisica

Nota: La distribución porcentual no suma $100 \%$, porque no se grafico Otra situación.

Fuente: INEGI, Censo de Población y Vivienda 2010.

Por otro lado, las entidades federativas con mayor población de adultos mayores económicamente activos son: Chiapas con un 42\%, le siguen en importancia Quintana Roo, Guerrero, Oaxaca y Tabasco, con 38.9, 37.8, 36.6 y 36.0\%, respectivamente, en este caso Baja California tiene un $30 \%$ y las entidades federativas con menor población de adultos mayores activos tenemos a Coahuila de Zaragoza con 24.7 y Nuevo León con un 23.5\%. 


\section{Cuadro 3. Distribución de la población de 65 años y más por grupo de edad y según actividad, por sexo, 2010.}

\begin{tabular}{|c|c|c|c|c|c|c|}
\hline \multirow{2}{*}{ México } & \multicolumn{2}{|c|}{ TRABAJA } & \multicolumn{2}{c|}{ NO TRABAJA } & \multicolumn{2}{c|}{ HOGAR } \\
\cline { 2 - 7 } & $\mathbf{H}$ & $\mathbf{M}$ & $\mathbf{H}$ & $\mathbf{M}$ & $\mathbf{H}$ & $\mathbf{M}$ \\
\hline $\mathbf{6 5 - 6 9}$ & 55.2 & 16.8 & 42.8 & 14.8 & 2.0 & 68.4 \\
\hline $\mathbf{7 0 - 7 4}$ & 43.1 & 11.9 & 54.4 & 19.2 & 2.5 & 68.9 \\
\hline $\mathbf{7 5 - 7 9}$ & 33.2 & 7.8 & 63.7 & 24.6 & 3.1 & 67.6 \\
\hline $\mathbf{8 0}$ y más & 18.5 & 3.9 & 77.7 & 42.1 & 3.8 & 54.0 \\
\hline
\end{tabular}

Fuente: Ham Chande, 2003, Censo de Información y Estadística 2010.

Podemos observar en la distribución de la población de 65 años, (Cuadro 3) que los hombres dentro el rango de 65-69 años continúan laborando y que disminuyo en el rango de los 80 años y más. No obstante, refleja su alta actividad y expresa su supuesta dependencia en la vejez. (Ham, 2003).

\section{Políticas Públicas de Pensiones y Jubilaciones}

Destaca el constante crecimiento demográfico y el esquema de beneficio definido que se maneja en 74 regímenes de los 105 que hay en el sistema de pensiones está ocasionando que éste sea insostenible, indica la Auditoría Superior de la Federación (ASF) en el reporte Evaluación de la Política Pública de Pensiones y Jubilaciones 2013. 
Cuadro 4. Evaluación de la política pública de pensiones y jubilaciones 2013.

\section{PESADO CUMPLIMIENTO}

Entre el 2004 y el 2013, el presupuesto ejercido en la política de pensiones y jubilaciones registró, en términos reales, un crecimiento promedio anual de $12.8 \%$, lo que significó que en este periodo se triplicaron los recursos para pensiones.

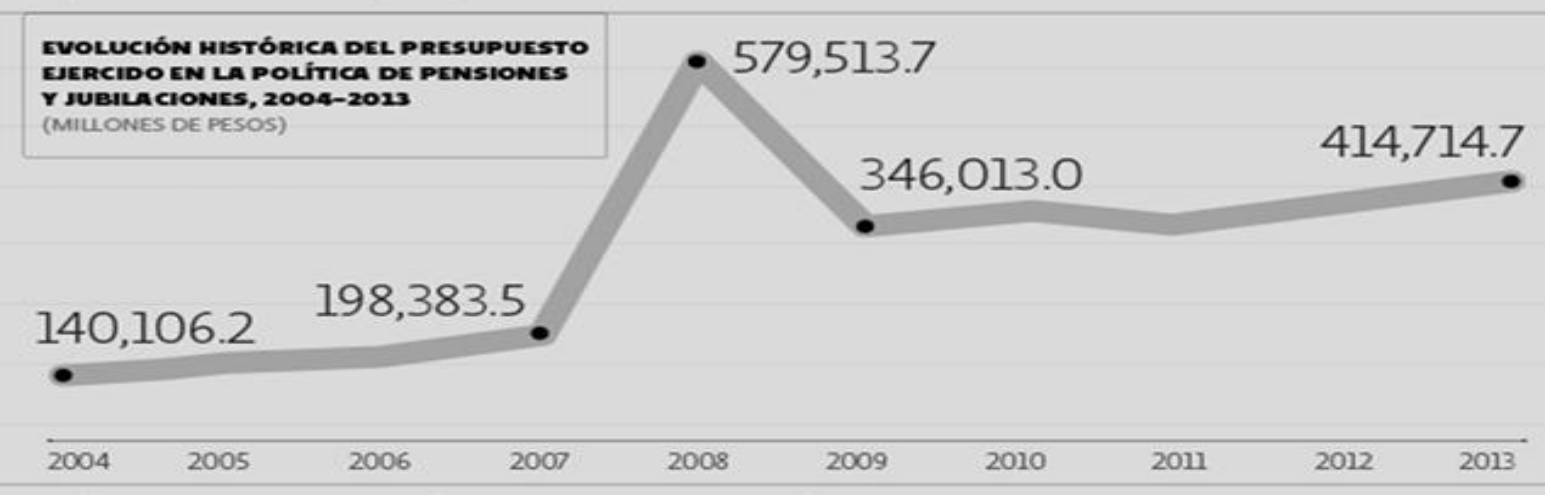

Fuente: Gráfico EE. Auditoría Superior de la Federación.

Del 2004 al 2013, el presupuesto ejercido en la política de pensiones y jubilaciones registró un crecimiento promedio anual de $12.8 \%$ en términos reales, al pasar de 140,106.2 millones de pesos en el 2004 a 414,714.7 millones de pesos en el 2013, (Cuadro4), lo que significó que en este periodo se triplicó el presupuesto ${ }^{7}$

Es necesario promover la actualización del sistema pensionario acorde a la realidad demográfica de los trabajadores al servicio del Estado y económica del país, considerando experiencias internacionales" En el 2013 significó 29.8\% de los ingresos tributarios, es decir 4.2 puntos porcentuales más que en el 2008 cuando la relación era de 25.6 por ciento.

La alta deuda en el pago de pensiones es un reflejo de que el sistema está mal diseñado y urge cambiarlo. Por cada millón de personas afiliadas al IMSS, al país le cuesta dos puntos

7 El Economista. El sistema de pensiones es insostenible: ASF, 3 agosto 2015. http://eleconomista.com.mx/finanzas-publicas/2015/02/18/sistema-pensiones-insostenible-asf 
porcentuales del PIB. (Cuadro 5). El sistema de pensiones representa un alto costo para el país, ya que la deuda en este rubro representa alrededor de $120 \%$ del Producto Interno Bruto

(PIB). ${ }^{8}$ Evaluación de la Política Pública de Atención a las Personas Adultas Mayores 1645 DGADDS.

\section{Cuadro 5. Programa Anual para la fiscalización superior de la cuenta pública 2014.}

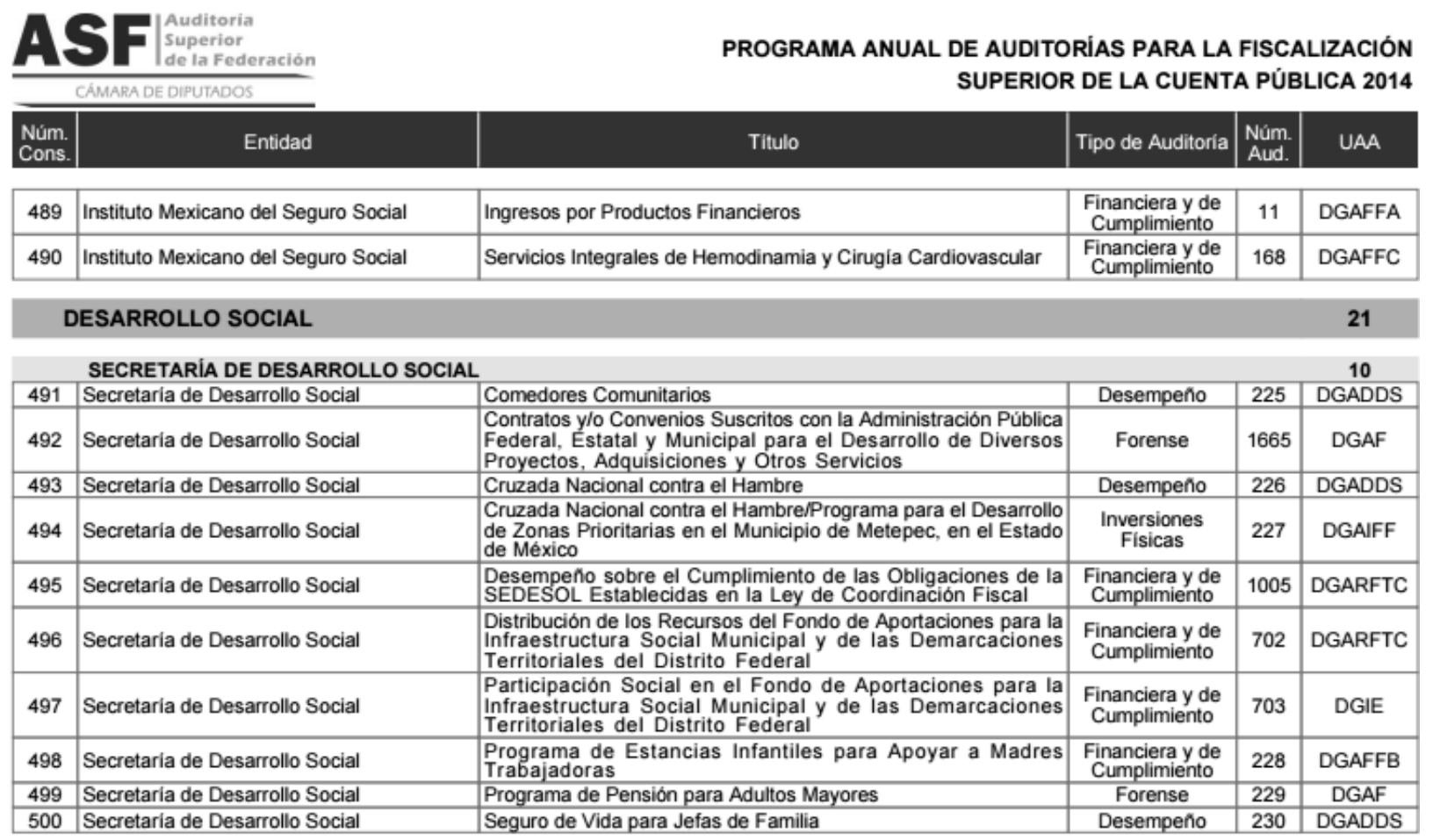

Fuente: Auditoría Superior de la Federación, 2014.

8 El Economista. Deuda en pago de pensiones representa $120 \%$ del PIB. 10 de febrero 2015 http://eleconomista.com.mx/sistema-financiero/2015/02/10/deuda-pago-pensiones-representa-120-pib 


\section{Cuadro 6. Integración de la muestra de pensiones y jubilaciones.}

(Cifras en miles de pesos)

\begin{tabular}{|c|c|c|c|}
\hline Delegación & Total ejercido & $\%$ & Muestra \\
\hline Oficinas Centrales & $988,651.6$ & $8.2 \%$ & $98,865.2$ \\
\hline Hidalgo & $3,057,716.2$ & $25.4 \%$ & $305,771.6$ \\
\hline Oaxaca & $4,844,575.9$ & $40.3 \%$ & $484,457.6$ \\
\hline San Luis Potosí & $3,145,193.6$ & $26.1 \%$ & $314,519.4$ \\
\hline Total & $12,036,137.3$ & $100.0 \%$ & $1,203,613.8$ \\
\hline
\end{tabular}

Fuente: Resumen de la emisión de pensiones pagadas y no pagadas a cargo del Gobierno Federal por periodo de enero a diciembre 2013 de la dirección de Finanzas del ISSSTE

Se constató que en 2013, la Secretaría de Hacienda y Crédito Público autorizó recursos al Instituto de Seguridad y Servicios Sociales de los Trabajadores del Estado (ISSSTE) para la partida presupuestal 4500 "Pensiones y Jubilaciones" (Cuadro 6) en el ámbito nacional para las 35 delegaciones y Oficinas Centrales, de las que se seleccionó un universo de 12,036,137.3 miles de pesos, que se integra por 988,651.6 miles de pesos, asignados a Oficinas Centrales; 3,057,716.2 miles de pesos, a la Delegación Hidalgo; 4,844,575.9 miles de pesos, a la Delegación Oaxaca; 3,145,193.6 miles de pesos, a la Delegación San Luis Potosí y los 110,631,119.9 miles de pesos restantes fueron asignados a las demás delegaciones. $^{9}$

Como objetivo de dicha auditoria es de fiscalizar la gestión financiera del régimen de jubilaciones y pensiones del instituto, en su carácter de administrador, respecto de sus operaciones y verificar que los recursos se otorgaron, calcularon, autorizaron, pagaron y registraron con transparencia, así como las acciones realizadas para afrontar sus obligaciones, de conformidad con las disposiciones legales y normativas.

\footnotetext{
${ }^{9}$ No. Auditoría: 469 Título Auditoría: Pensiones y Jubilaciones Año Cuenta Pública: 2013 Entidad Fiscalizada: Instituto de Seguridad y Servicios Sociales de los Trabajadores del Estado Tipo Auditoría: Financiera y de Cumplimiento Sector: Entidades No Coordinadas Sectorialmente Entidad Federativa: Oaxaca
} 
Se observó discrepancia entre la normativa institucional y la actividad real de las áreas operativas, como es el caso de la documentación que se requiere para otorgar pensiones y prestaciones complementarias, que actualmente no es necesaria por estar registrada en el sistema de pensiones antes de su validación, o por la circular número SP-00020/11 del 15 de agosto de 2011, que en la práctica sustituyó al procedimiento 9, “Trámite de Indemnización Global" del Manual de Procedimientos de Delegaciones, que establecía formatos que ya no son utilizados, como la solicitud de pensiones (formato M-22 y M-23), que difiere del que se genera en forma automática por el Sistema Administrador de Otorgamiento (SAO).

\section{Adultos Mayores Ocupados en México}

En lo referente a los adultos mayores de 60 años y más ocupada se tiene por entidad federativa que Chiapas con un 98.3\%, Yucatán y Campeche en un 98\%, Oaxaca en $97.5 \%$ en comparación con Baja California con un $95 \%$ y los que menos adultos ocupados tienen son Chihuahua y Aguascalientes con 94.2\%.

Los adultos mayores entre los 60-64 años, donde se sobreentiende que el estado de las capacidades físicas e intelectuales aún permiten el desarrollo de tareas laborales, destacando asalariados y empleadores, en el sector primario $^{10}$ el cual ha experimentado durante el periodo de 1990 al 2010, un franco descenso de casi 15.0 puntos porcentuales, la población ocupada disminuye drásticamente conforme se incrementa su edad.

Ahora bien, el CONEVAL, (Cuadro 7). En nota informativa del 28 de agosto del 2011. (Día de la población adulta mayor), comunicó en el 2010, que 3.5 millones de personas de 65 se

10 Incluye agricultura, ganadería, aprovechamiento forestal, caza y pesca. INEGI. Censo de Población y Vivienda 2010, cuestionario ampliado. 
encontraban en pobreza ${ }^{11}$, de las cuales 800 mil se encontraron en pobreza extrema ${ }^{12}$. El número de personas mayores de 65 años con un ingreso inferior a la línea de bienestar en 2008 era de 3.5 millones, en 2010 aumentó a 3.9 millones. El porcentaje de la población mayor de 65 años con un ingreso inferior a la línea de bienestar mínimo ${ }^{13}$ pasó de $18.6 \%$ en 2008 a $19.2 \%$ en 2010.

Cuadro 7.

\section{Población adulta mayor, 2010}

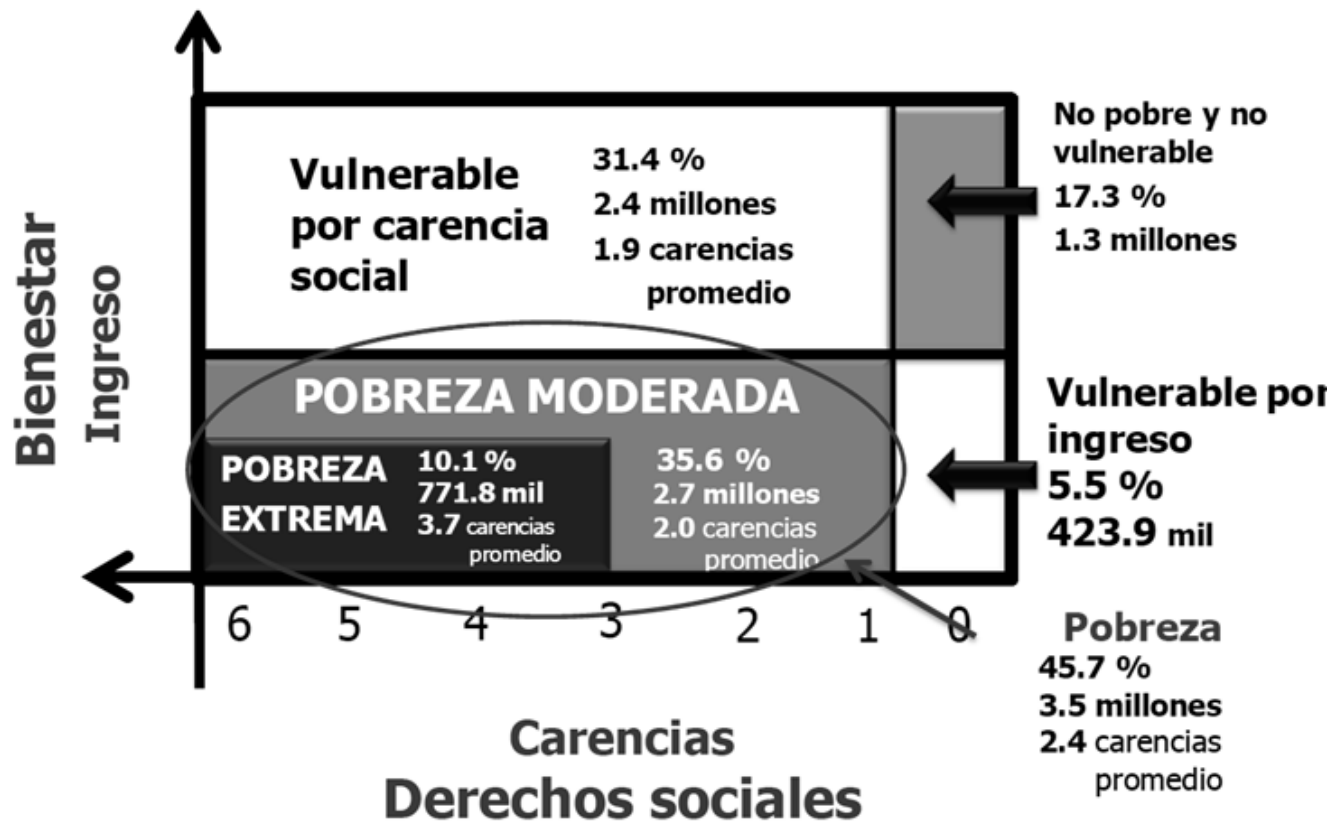

Fuente: estimaciones del CONEVAL con base en el MCS-ENIGH 2010.

\footnotetext{
${ }^{11}$ Pobreza. Una persona se encuentra en situación de pobreza cuando tiene al menos una carencia social (en los seis indicadores: rezago educativo, acceso a servicios de salud, acceso a la seguridad social, calidad y espacios de la vivienda, servicios básicos en la vivienda y acceso a la alimentación), y su ingreso es menor que el valor de la Línea de Bienestar Económico. (CONEVAL 2014).

${ }^{12}$ Pobreza extrema. Una persona se encuentra en situación de pobreza extrema cuando tiene tres o más carencias sociales de los indicadores y su ingreso es menor que el valor de la Línea de Bienestar Mínimo (CONEVAL 2014).

${ }^{13}$ Línea de Bienestar Mínimo. Valor monetario de la canasta alimentaria básica. Es un subconjunto de la Línea de Bienestar (CONEVAL 2014).
} 


\begin{abstract}
Asistencia Social
Los elementos que asocian a este concepto son las libertades individuales, la igualdad, derechos sociales, responsabilidad pública, la justicia social, bienestar, vulnerabilidad, marginación y pobreza, ver cuadro 8, (Guadarrama 2001: 89-90). Se puede hablar de los débiles sociales y económicos, y pasa de la beneficencia al concepto de asistencia pública que realiza el Estado.

En la actualidad en México la administración asistencial le corresponde al Estado y se ejerce a través de tres entidades, es decir dos secretarías, las de Salud y Desarrollo Social, y por el organismo descentralizado del Sistema Nacional DIF, donde algunas funciones también son acatadas por las procuradurías sociales a nivel estatal y municipal de las entidades federativas.
\end{abstract}

En México la asistencia social es una expresión de solidaridad humana que se manifiesta en la familia, en la sociedad y en el ámbito del Estado. Su importancia es tal que se regula en la Ley General de Salud, la Ley de Asistencia Social y la Ley de los Derechos de las Personas Adultas Mayores. Es el conjunto de acciones tendientes a modificar y mejorar las circunstancias de carácter social que impidan el desarrollo integral del individuo, así como la protección física, mental y social de personas en estado de necesidad, desprotección o desventaja física y mental, hasta lograr su incorporación a una vida plena y productiva. La asistencia social comprende acciones de promoción, previsión, prevención, protección y rehabilitación.

Existen 8.5 millones de personas que no tienen carencias, pero son vulnerables por ingreso. - 43.9 millones de personas (36.6\%) son pobres, lo que implica que están debajo de la línea de bienestar económico y cuentan con casi dos carencias en promedio. 
En total, 55.3 millones de personas viven en situación de pobreza y carecen de derechos sociales. El CONEVAL indica que en México 43 por ciento de los adultos mayores se encuentran en situación de pobreza multidimensional.

En México la pobreza multidimensional se define como la situación de una persona cuando presenta carencia de al menos uno de sus derechos relacionados con el desarrollo social, y si además sus ingresos son insuficientes para adquirir los bienes y servicios que requiere para satisfacer sus necesidades.

\section{Cuadro 8.}

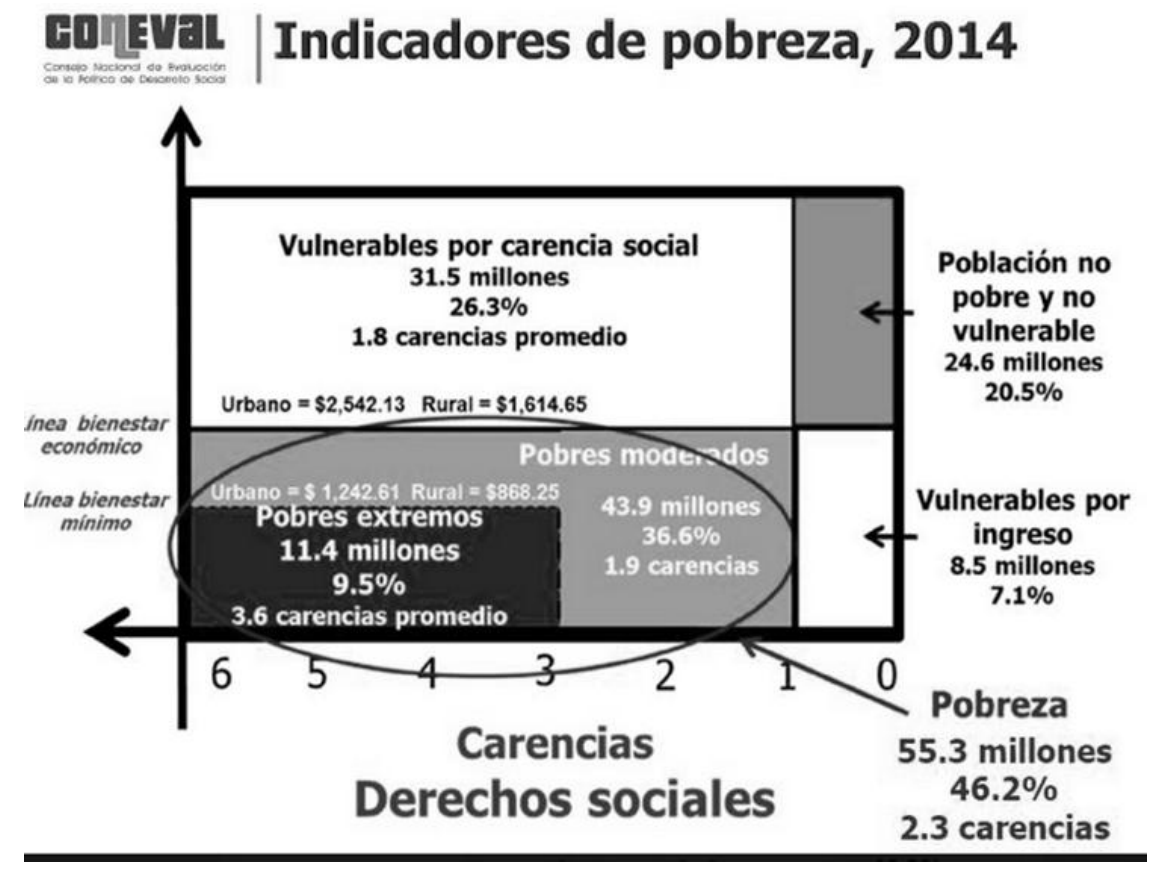

Fuente: CONEVAL 2014, Informe de publicación 2015.

Además, la mayoría son mujeres. Sólo 2 de cada 10 adultas mayores de 65 años cuenta con una pensión, 2 de cada 10 adultos mayores pueden solventar sus gastos, el resto se encuentra en pobreza: 3.5 millones siendo el $45.7 \%$, en pobreza moderada; 2.7 millones siendo el 
$36.6 \%$, con 800 mil en pobreza extrema siendo el 10.1; por otro lado hay otro 31.4 por ciento de mexicanos de más de 65 años que son vulnerables y sufren dos carencias y, 77.1 por ciento (5.9 millones) tiene al menos una privación social", dice reporte fechado en febrero 2015 en la Cámara de Diputados.

Según el Instituto Nacional de las Personas Adultas Mayores, tres de cada cinco ancianos sufren violencia dentro de la familia. La Encuesta Nacional sobre Discriminación en México 2010 muestran que en el país las personas adultas mayores integran el cuarto grupo de población vulnerable; y sólo en 17 de las 32 entidades federativas hay legislaciones locales de no discriminación a este sector

\section{Normatividad de la Asistencia Social}

La reglamentación se dispersa en distintas leyes y códigos, circunstancia natural si se toma en cuenta que el derecho social, ${ }^{14}$ tiene su raíz legal en la norma constitucional, el derecho a la educación (artículo 3o.) la protección a la salud, el derecho a la vivienda (artículo 4o.), el derecho y la libertad al trabajo (artículo 5o.), las facilidades para organizar y expandir la actividad económica del sector social (artículo 25), la protección de los derechos de los trabajadores (artículo 123).

Con referencia a los establecimientos de asistencia social temporal para adultos mayores se debe contar preferentemente con el siguiente personal: responsable sanitario del establecimiento, trabajador social, terapeuta ocupacional, promotor de la salud, cocinera, intendente, cuidador y vigilante, éste las 24 horas del día.

\footnotetext{
14 Mediante reformas y adiciones que se le imprimieron al artículo $4^{\circ}$ de la Constitución Política Federal, el 3 de febrero de 1983, se elevó a rango constitucional el derecho a la protección de la salud como una garantía individual y social de los mexicanos, al señalar que: "toda persona tiene derecho a la protección de la salud. La ley definirá las bases y modalidades para el acceso a los servicios de salud y establecerá la concurrencia de la Federación y las Entidades federativas en materia de salubridad general, conforme a lo que dispone la fracción XVI del artículo 73 de esta Constitución...
} 
Las instituciones de asistencia social de los sectores público, social y privado, por medios propios o a través de terceros, serán responsables de capacitar al personal en temas relacionados con el proceso de envejecimiento y la vejez y educación continua en la materia, preferentemente una vez al año.

NORMA Oficial Mexicana NOM-031-SSA3-2012, Asistencia Social. Prestación de Servicios de Asistencia Social para Adultos y Adultos Mayores en Situación de Riesgo y Vulnerabilidad.

Esta Norma tiene por objeto establecer las características de funcionamiento, organización e infraestructura que deben observar los establecimientos de los sectores público, social y privado, que presten servicios de asistencia social a personas adultas y adultas mayores en situación de riesgo y vulnerabilidad. Donde las siguientes instituciones acordaron estas funciones de organización como obligatorias a todo el territorio nacional en los establecimientos de asistencia social y en donde se puede consultar las siguientes normas.

Dirección General de Rehabilitación y Asistencia Social

Centro Nacional Modelo de Atención, Investigación y Capacitación Gerontológico >Arturo Mundet<

Dirección General Jurídica y Enlace Institucional

Sistema Estatal DIF Campeche

Sistema Estatal DIF Chiapas

Sistema Estatal DIF del Gobierno del Distrito Federal

Sistema Estatal DIF Estado de México

Sistema Estatal DIF Guerrero

Sistema Estatal DIF Hidalgo

Sistema Estatal DIF Jalisco

Sistema Estatal DIF Michoacán 
Sistema Estatal DIF Morelos

Sistema Estatal DIF Nuevo León

Sistema Estatal DIF Oaxaca

Sistema Estatal DIF Puebla

Sistema Estatal DIF Querétaro

Sistema Estatal DIF Quintana Roo

Sistema Estatal DIF San Luis Potosí

Sistema Estatal DIF Sonora

Sistema Estatal DIF Tlaxcala

Sistema Estatal DIF Veracruz

Sistema Estatal DIF Yucatán

Norma Oficial Mexicana NOM-233-SSA1-2003, Que establece los requisitos arquitectónicos para facilitar el acceso, tránsito, uso y permanencia de las personas con discapacidad en establecimientos de atención médica ambulatoria y hospitalaria del Sistema Nacional de Salud. (Renovada).

Norma Oficial Mexicana NOM-168-SSA1-1998, Del expediente clínico.

En la Norma Oficial Mexicana NOM-167-SSA1-1997, para la prestación de servicios de asistencia social para menores y adultos mayores, en la cual colaboraron:

Secretaría de Salud.

Secretaría de Comercio y Fomento Industrial.

Secretaría de Educación Pública.

Secretaría de Gobernación.

Secretaría de la Defensa Nacional.

Sistema Nacional para el Desarrollo Integral de la Familia.

Organización Panamericana de la Salud.

Gobierno del Distrito Federal.

Procuraduría General de Justicia del Distrito Federal. 
Instituto Mexicano del Seguro Social.

Instituto de Seguridad y Servicios Sociales de los Trabajadores del Estado.

Instituto Nacional de la Nutrición >Salvador Zubirán<.

Instituto Nacional de la Senectud.

Instituto Nacional para la Rehabilitación de Niños Ciegos y Débiles Visuales.

Hospital para Enfermos Crónicos >Gustavo Baz<.

Junta de Asistencia Privada.

Petróleos Mexicanos.

Universidad Nacional Autónoma de México.

En nuestro país la Asistencia Social es una expresión de solidaridad humana que se manifiesta en la familia, en la sociedad y en el ámbito del Estado, cuyas acciones se han caracterizado por la operación de programas circunstanciales que limitan el desarrollo de la asistencia social, amén que el retardo en la solución a los problemas más urgentes y el surgimiento de nuevas necesidades incrementan los rezagos, principalmente en los sectores más pobres de la sociedad, en los grupos más vulnerables como son los menores y adultos mayores en condición de orfandad, abandono, rechazo social y maltrato físico y mental.

Tiene como objeto establecer los procedimientos para uniformar principios, criterios, políticas y estrategias en la prestación de servicios y desarrollo de actividades en materia de asistencia social a menores y adultos mayores.

Se pretende en establecimientos para la atención del adulto mayor: Promover el bienestar presente y futuro de los adultos mayores. Mejorar la prestación de servicios de asistencia social. Estimular la interacción del adulto mayor. Estimular la participación social. Realizar acciones que promuevan la participación en actividades ocupacionales y recreativas. Fomentar el respeto a la persona, a sus derechos humanos.

Es aplicable en todas las dependencias y entidades de la administración pública, tanto federal como local y las personas física o moral de los sectores social y privado que conforman el Sistema Nacional de Salud, ver cuadro 5. Para su aplicación NOM-001-SSA2-1993, que 
establece los Requisitos Arquitectónicos para Facilitar el Acceso, Tránsito y Permanencia de los Discapacitados a los Establecimientos de Atención Médica del Sistema Nacional de Salud.

Se define como adulto mayor en estado de abandono, aquel que presenta cualquiera de las características siguientes: carencia de familia, rechazo familiar, maltrato físico o mental, carencia de recursos económicos.

A continuación, se proporcionan conceptos relacionados con instituciones que atienden a este sector poblacional: Casa hogar para adultos mayores, son establecimientos de asistencia social donde se proporciona a los adultos mayores atención integral mediante servicios de alojamiento, alimentación, vestido, atención médica, trabajo social, actividades culturales, recreativas, ocupacionales y psicológicas.

Centro de día, es el establecimiento público, social o privado que proporciona alternativas a los adultos mayores para la ocupación creativa y productiva del tiempo libre mediante actividades culturales, deportivas, recreativas y de estímulo, donde se promueve tanto la dignificación de esta etapa de la vida, como la promoción y autocuidado de la salud.

Dar seguimiento a estas normas corresponde a la Secretaria de Salud y a los Gobiernos Estatales en el ámbito de su respectiva competencia. Publicado en el Diario de la Federación el 17 de noviembre 1999.

En el informe del Centro de Estudios de las Finanzas Públicas se observa que, de acuerdo a la Encuesta Nacional de Ocupación y Empleo, en el cuarto trimestre del 2012, la cobertura en seguridad social de la población ocupada fue alrededor del 36\%, es decir 6 de cada 10 personas ocupadas no cotizan en alguna institución de salud. 
Cuadro 9. Población cubierta por institución de Salud en México, 2010-2011.

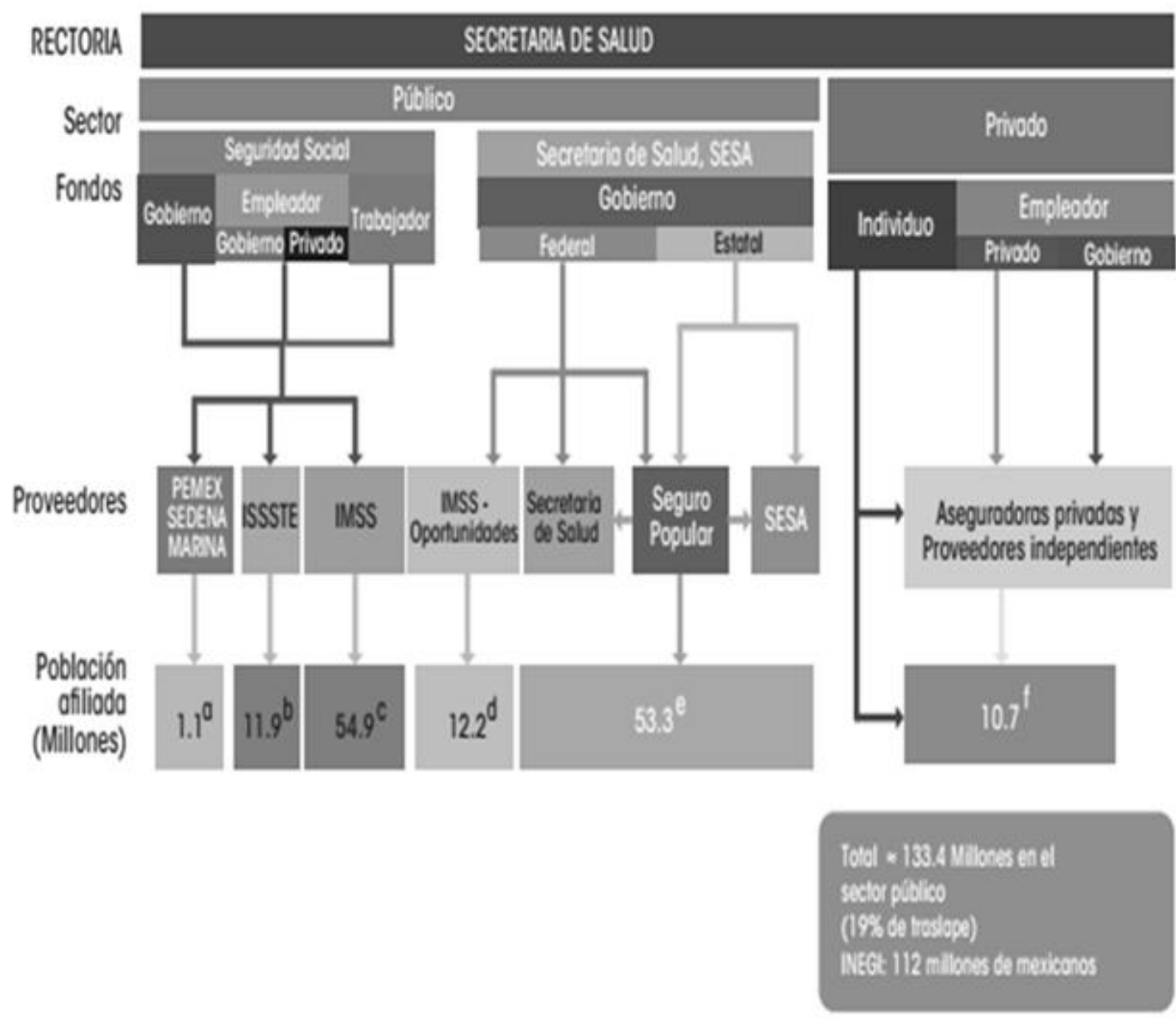

Fuente: Datos de ENGH (2010), Portal ISSSTE (2010), Norma Oficial Mexicana (2011) y portal de la Comisión Nacional de Seguros. 
Es importante reconocer que en el 2012 existe un avance importante en la cobertura del Seguro Popular, que no se ve reflejado en las cifras anteriores (Cuadro 9). El Artículo 123 de la Constitución, relativo al trabajo.

En éste se definen las coberturas sociales mínimas que deben otorgarse a los trabajadores y sus familias. La Ley de Seguridad Social (LSS) establece que la finalidad de ésta es garantizar el derecho a la salud, la asistencia médica, la protección de los medios de subsistencia y los servicios sociales necesarios para el bienestar individual y colectivo (CONEVAL, 2010a: 5556).

Sin embargo, los sistemas de seguridad social contributiva creados hace más de 70 años para dar cumplimiento a este artículo constitucional (principalmente IMSS e ISSSTE) cubren actualmente una fracción minoritaria de la población, por lo que se han introducido otros instrumentos de gasto social para ofrecer esta protección a la población no asegurada, como el Seguro Popular y el programa 70 y Más.

\section{Adulto Mayor en Baja California}

El Consejo Nacional de Población CONAPO 2016, nos presenta que en Baja California su población de 65 años y más representa un 5.4\% de su población, es decir que 5 de cada 100 habitantes tienen 65 años y más, se estima que para el 2030 aumente al 9.2\%.

La Encuesta Intercensal 2015, realizada por Instituto Nacional de Estadística y Geografía INEGI, presentó que $9.3 \%$ de las personas mayores de 65 años y más son analfabetas, además el $50.3 \%$ se encuentran casados.

Con respecto al II trimestre del 2016 en Baja California, la Encuesta Nacional de Ocupación y Empleo ENOE, elaborada por INEGI, arrojó los siguientes datos de la Población Económicamente Activa PEA, en la población de 65 años y más un total de 40 mil 462 
habitantes, mientras que la población de 60 años y más No Económicamente Activa registró 232 mil 018 habitantes.

\section{Conclusiones}

La relevancia que adquiere este trabajo se debe a que en México se tiene una población que oscila en los 10.5 millones de Adultos Mayores, de los cuales 2.8 millones tienen alguna limitante en la actividad, lo cual implica que éste grupo etario se observa muy desfavorecido y en algunas ocasiones cae en vulnerabilidad.

Para poder atacar el factor de vulnerabilidad en el que cae la población en esta situación debemos recordar que los gobiernos han empleado estrategias enfocadas en la asistencia social, que es un principio del Estado, pero no le es exclusiva, ni como función ni como obligación. Puesto que es un derecho para quien se encuentra en estado de necesidad o de vulnerabilidad.

Se requiere de Políticas Públicas que permitan que su población participe en el incremento del Producto Interno Bruto (PIB), por medio del ahorro y que le permita resolver el costo de vida, con atención precautoria en la atención social y médica para los futuros adultos mayores, con lo cual se pudiese limitar la inclusión de una mayor cantidad de personas en este rubro de vulnerabilidad.

Una estrategia que pudiese emplearse para disminuir el factor limitante de este grupo social es que el sistema de pensiones debe basarse en un modelo nacional, ya que en el país existen más de 100 esquemas diferentes de pensiones.

Ante el crecimiento de la esperanza de vida, los trabajadores deben laborar más años para poder sostener el financiamiento de su pensión y deben ahorrar más si desean que su pensión se asemeje a su último salario. 
Se considera que mientras no exista un esquema de cuentas individuales y contributivas, donde los trabajadores sean y se hagan responsables de sus ahorros, la deuda en el pago de pensiones continuará creciendo. Se tiene como reto la modificación de los esquemas de pensiones donde se pueda equilibrar lo político, económico y lo social.

Por otro lado, se requiere darle seguimiento de acuerdo con la Ley Federal para Prevenir y Erradicar la Discriminación, donde se entiende por discriminación toda distinción, exclusión, restricción o preferencia que, por acción u omisión, con intención o sin ella, no sea objetiva, racional ni proporcional y tenga por objeto o resultado obstaculizar, restringir, impedir, menoscabar o anular el reconocimiento, goce o ejercicio de los derechos humanos y libertades, cuando se base en uno o más de los siguientes motivos: el origen étnico o nacional, el color de piel, la cultura, el sexo, el género, la edad, las discapacidades, la condición social, económica, de salud o jurídica, la religión, la apariencia física, las características genéticas, la situación migratoria, el embarazo, la lengua, las opiniones, las preferencias sexuales, la identidad o filiación política, el estado civil, la situación familiar, las responsabilidades de estas, el idioma, los antecedentes penales o cualquier otro motivo.

Es conveniente observar y dar seguimiento a la población adulta que se encuentra en los rangos entre los 40 y 59 años, dado que pronto formara parte de la población de Adultos Mayores, observación que ya advirtió el INEGI con 17 entidades federativas que superaron la tasa de crecimiento poblacional.

Asimismo, se presenta una falta de incentivos para la inversión privada en la generación de tecnologías para la atención de la vulnerabilidad social. En las instituciones tenemos fractura del sistema nacional de asistencia social. Además, desestructuración del Sistema Nacional DIF y propuestas estatales desvinculadas.

Finalmente coincidimos con el argumento de Avishai Margali (1996) "La sociedad caritativa se basa en el principio de benevolencia, y la sociedad del bienestar en el principio del derecho. 
Una sociedad que asiste a los necesitados por el derecho que éstos tienen a la asistencia es menos humillante, en principio, que una sociedad basada en la benevolencia". 


\section{Referencias}

Auditoria Superior de la Federación ASF, Programa anual de auditorías para la fiscalización $\begin{array}{lllll}\text { superior de la } & \text { cuenta } & \end{array}$ http://www.asf.gob.mx/uploads/54_Informes_y_publicaciones/PAAF_CP14_Por_E ntidad_Fiscalizada_20150630.pdf .

Alwanget et al, La vulnerabilidad frente al riesgo (2011). s/d

ASF. No. Auditoría: 469 Título Auditoría: Pensiones y Jubilaciones Año Cuenta Pública: 2013 Entidad Fiscalizada: Instituto de Seguridad y Servicios Sociales de los Trabajadores del Estado Tipo Auditoría: Financiera y de Cumplimiento Sector: Entidades No Coordinadas Sectorialmente Entidad Federativa: Oaxaca (2003). http://www.asf.gob.mx:8081/Informe.aspx

Cardona, O. Estimación Holística del riesgo sísmico utilizando sistemas dinámicos complejos (2001).

Centro de Estudios de las Finanzas Públicas CEFP. (2013) Indicadores sobre seguridad social en México.

http://www.cefp.gob.mx/indicadores/gaceta/2013/iescefp0152013.pdf

Censo de Población y Vivienda (2010). Perfil sociodemográfico de adultos mayores / Instituto Nacional de Estadística y Geografía. -- México: INEGI, 2014.

Chambers, R. (1989) Vulnerability, coping and policy, IDS Bulletin No. 20, Institute of Development Studies of Development Studies, Sussex.

Consejo Nacional de Evaluación de la Política de Desarrollo Social. (2015). Informe de Evaluación de la Política de Desarrollo Social en México 2014 México, DF: CONEVAL.

Consejo Nacional de Población CONAPO 2016. Adultos Mayores 2016-2030

http://www.copladebc.gob.mx/publicaciones/2016/ADULTOS\%20MAYORES\%2020162030.pdf

Estudios económicos de la OCDE MÉXICO ENERO 2015 VISIÓN GENERAL

file://C:/Users/UABC/Desktop/Envejecimiento\%202015/Mexico-Overview2015\%20Spanish.pdf Consultado Agosto 42015.

Guadarrama, G, Entre la Caridad y el Derecho. Un estudio sobre el agotamiento del Modelo Nacional de Asistencia social. El Colegio Mexiquense, A.C./Consejo Estatal de Población, Zinacantepec, México. (2001)

Ham, R. Envejecimiento en México: el siguiente reto de la transformación demográfica. El Colegio de la Frontera Norte. Tijuana. (2003).

Ley De Los Derechos De Las Personas Adultas Mayores, publicada en el Diario Oficial de la Federación el 25 de junio de 2002. Última reforma publicada DOF 25-04-2012. http://www.diputados.gob.mx/LeyesBiblio/pdf/245.pdf Consultada 27 de Julio 2015. 
NORMA OFICIAL MEXICANA NOM-031-SSA3-2012, Asistencia social. Prestación de servicios de asistencia social a adultos y adultos mayores en situación de riesgo vulnerabilidad

.http://dof.gob.mx/nota_detalle.php?codigo=5267965\&fecha=13/09/2012 Consultado 29 de Julio 2015.

NORMA OFICIAL MEXICANA NOM-233-SSA1-2003, Que establece los requisitos arquitectónicos para facilitar el acceso, tránsito, uso y permanencia de las personas con discapacidad en establecimientos de atención médica ambulatoria y hospitalaria del Sistema Nacional de Salud.

http://www.salud.gob.mx/unidades/cdi/nom/233ssa103.html Consultado 27 de Julio 2015.

NORMA OFICIAL MEXICANA NOM-168-SSA1-1998, del expediente clínico. http://www.salud.gob.mx/unidades/cdi/nom/168ssa18.html Consultado 27 de Julio 2015.

NORMA OFICIAL MEXICANA NOM-167-SSA1-1997, para la prestación de servicios de asistencia social para menores y adultos mayores.

http://www.salud.gob.mx/unidades/cdi/nom/167ssa17.html Consultado 27 de Julio 2015.

Holzmann, R. y S. Jorgensen. Manejo social del riesgo: un nuevo marco conceptual para la protección social y más allá. Serie de Documentos de Discusión sobre la Protección Social. Washington: Banco Mundial. (2000)

Kabeer, N. Safety Nets and Opportunity Ladders: Addressing Vulnerability and Enhancing Productivity in South Asia. Working Paper 159. Brighton: Institute of Development Studies, University of Sussex. (2002).

Susman, P., O'Keefe, P. and Wisner, B. Global disasters: A radical interpretation pp. 26483 in Hewitt. (1984)

Margalit, A. The Decent Society. Harvard University Press. (1996)

http://www.hup.harvard.edu/catalog.php?isbn=9780674194373, Consultado 2 julio 2015.

Organización Internacional del Trabajo OIT (1984). Normas internacionales del trabajo, Memoria del Director General (I), Conferencia Internacional del Trabajo, 70. ${ }^{a}$ reunión, Ginebra, 1984

Rodríguez V., J., Vulnerabilidad y grupos vulnerables: un marco de referencia conceptual mirando a los jóvenes, Serie Población y Desarrollo, N17, CELADE, Santiago de Chile. (2001)

Ruiz, N., "La definición y medición de la vulnerabilidad social. Un enfoque normativo" Investigaciones Geográficas, No. 777, México. (2012)

Wisner, B., Blaikie, P., Cannon, T., Davis, I., "At risk: natural hazards, people's vulnerability and disasters", Routledge, Londres. (2004). 